\title{
Histopathologic Characteristics of Invasive and Non-invasive Ductal Tumors have Relationship with Different Phenotypes of ER / PR Receptors in Breast Cancer Patients
}

\author{
Rahim Golmohammadi ${ }^{1}$, Mohammad Reza Mohajeri², AlirezaMosavi Jarrahi ${ }^{3}$, \\ Ali Reza Moslem ${ }^{4}$, Akbar Pejhan ${ }^{5}$, Ali Gohari ${ }^{6}$
}

${ }^{1}$ Department of Anatomy, Faculty of Medicine, Sabzevar University of Medical Sciences, Sabzevar, Iran. ${ }^{2}$ Department of Pathology, Shahid Sadoughi Hospital, Isfahan, Iran. ${ }^{3}$ Faculty of Medical School, Shahid Beheshti University of Medical Sciences, Tehran, Iran. ${ }^{4}$ Department of Anesthesiology, Faculty of Medicine, Sabzevar University of Medical Sciences, Sabzevar, Iran. ${ }^{5}$ Faculty of Medicine, Sabzevar University of Medical Sciences, Sabzevar, Iran. ${ }^{6}$ Faculty of Medicine, Sabzevar University of Medical Sciences, Sabzevar, Iran.

\begin{abstract}
Objective: Contradictory reports have been published regarding the expression levels of the hormone receptors of estrogen and progesterone (ER / PR) and theirclinical importance in diagnosis of breast cancer. The aim of this study was to evaluate the relationship between pathological features of invasive and non-invasive ductal tumors by different ER / PR phenotypes. Methods: This descriptive-analytical study was performed on 74 specimens of breast cancer referred to Isfahan Hospitals for diagnosis between 2015 - 2018. After fixation of the specimens in formalin, tissue passage, cross section and $\mathrm{H} / \mathrm{E}$ staining, the specimens were divided into two groups: non- invasive and Invasive ductal Carcinoma. After removing of mask, expression of different ER / PR phenotypes was performed using primary monoclonal antibody and immunohistochemically methods. Results: From 74 malignant specimens, $61(82.4 \%)$ were in the category of invasive ductal tumors and 13 cases $(17.6 \%)$ were in the category of non-invasive ductal tumors. Out of 73 patients with positive ER or PR phenotype 47 samples (63.5\%) had ER + / PR + phenotypes, 6 samples had (8.1\%) ER+/ PR -phenotype, 20 samples (27\%) had ER- / PR + phenotype and only one sample (1.4\%) had the ER- / PR-phenotype and was in the category of invasive ductal tumors. There was not detected ER- / PR- phenotype expression in non-invasive ductal tumor. Further analysis showed that there were not significant difference between ER / PR phenotype and tumor stage $(\mathrm{p}=0.36)$ or with tumor Grade $(\mathrm{P}=0.38)$, high age of menopause or post menopause $(\mathrm{P}>0.05)$. Conclusion: Our data shows that expression of ER- / PR- phenotype only was detected in invasive ductal tumor. It is thought that the tumor type maybe affects the expression of different types of ER / PR hormone receptor phenotypes in breast cancer patients.
\end{abstract}

Keywords: Breast Neoplasm- ER / PR receptor expression- Grade- Stage- Tumor type

\section{Introduction}

Breast cancer is the second deadliest cancer in women and on the Asian continent shows an increasing incidence of malignancy in recent years [1]. Recent reports on breast cancer show that about half a million women are dying of this disease in worldwide each year [2]. The Sharma R report shows that the number of breast cancer patients was 1.7 million in 2016 , with the highest prevalence being reported in the Netherlands (117.2 per 100,000). The rate was 35.4 per 100,000 women in Afghanistan [3]. These findings show that prevalence of breast cancer is higher in developed countries than undeveloped countries. Breast cancer is the most common cancer between Iranian women and the prevalence of breast cancer is on average 22.6 women per $100,000[4,5]$.

Corresponding Author:

Dr. Ali Gohari

Department of Biochemistry, Faculty of Medicine, Sabzevar University of Medical Sciences, Sabzevar, Iran.

Email: gohari.biochem@gmail.com 
Breast cancer is mainly seen in both invasive and non-invasive forms. Invasive ductal carcinomas that account for about 70 to 85 percent of breast cancers include invasive ductal carcinoma no special type (NST) and invasive lobular carcinoma (ILC or NLC). Non-invasive carcinomas that account for about 15 to 30 percent of the remaining breast cancer include Ductal Carcinoma in situ (DCIS) and Lobular Carcinoma in situ (LCIS) [6].

The mean age of breast cancer is different in various geographical regions, which are subjected to different factors such as stress, environmental factors, epigenetic and genetic factors [7].

Genetic factors can be evaluated from two aspects in the process of progression of breast cancer.

The first group of genes being expressed with a good prognosis of the disease, while the lack of their expression causes a poor prognosis of the disease. In this group, genes such as P53, PTEN and several other markers can be mentioned [8-11]. The second group of genes being expressed highly and malignant cells invade to adjacent tissues, lymph nodes or distant organs. These genes can recurrent disease and results a poor prognosis in breast cancer $[12,13]$.

Gene expression of ER+ / PR+ is very important in prognosis and prediction of breast cancer patients [14]. There are a few reports regarding relationship between these hormone receptors (PR / ER) and grade or stage tumor from different regions of the world. The study of Gahlaut $\mathrm{R}$ et al indicates that there is a statistically significant relationship between grade and these hormone receptors expression [15], However, the study by Rauscher GH et al indicates that there is no any significant relationship between ER / PR expression with grade and tumor stage. On the other hand, it has been reported that deaths from breast cancer in black women with positive ER / PR are approximately four times more than similar white women. This study shows that positive ER / PR have not the same effect on prognosis and progression of the disease in the two groups of black and white women [16]. There are different reports on regarding the expression level of positive ER / PR receptors from different geographical locations. For example, it has been reported that positive ER / PR expression is 60 to $70 \%$ in India [17], although in other parts of the world have been reported $75 \%$ or even more [18]. It seems that, geographic location as an effective factor may play a role in the expression of ER + / PR hormone receptors by unknown pathway. There have not any complete reports on investigation the relationship between four different forms of the ER / PR phenotype with invasive and non-invasive duct tumors or with pre-menopausal and post-menopausal in breast cancer patients from center of Iran. Therefore, this study was designed to investigate the relationship between these two types of invasive and non-invasive ductal tumors with expression of different phenotypes of ER / PR hormone receptors simultaneously with pathologic features in breast cancer patients in center of Iran.

\section{Materials and Methods}

\section{Collection and preparation of samples}

This descriptive-analytical study was performed on 74 breast cancer patients referred to Isfahan hospitals in central of Iran during 2015-2018. This study was conducted according to the guidelines of the Ethics Committee of Isfahan University of Medical Sciences, Iran. Samples were collected before patients received radiotherapy or chemotherapy. Adjacent normal tissue was used as control sample. The tissue preparation steps are summarized as follows: fixation of the specimens in $10 \%$ formalin, tissue processing, paraffin embedding of the specimens, preparation of 4 micron slices of all specimens using Litzerotary microtome and finally staining with hematoxylin and eosin. Tumors were divided into Grades 1-3, according to the diagnosis of malignancy by two pathologists (double blind) and tissue grade was determined based on mitosis, polymorphism and presence or absence of gland in the specimens. Tumor stages were determined by routine staging from stage zero (in situ carcinoma) to stage 4 (metastasis). The specimens were divided into invasive and non-invasive groups according to pathological methods $[19,20]$.

\section{Evaluation of ER/PR Receptor Gene Expression by Immunohistochemistry}

The expression of ER and PR genes were evaluated by immunohistochemistry. The sequence of immunohistochemical procedures may be summarized as follows: Temperature and antibody concentration used according to the kit instructions: Microwave and citrate buffer at $100^{\circ} \mathrm{C}$ for 20 minutes were used to detect the location of the antigenic markers of the samples. In order to inhibit endogenous peroxidase activity, the slides were incubated in 3\% hydrogen peroxide solution for $30 \mathrm{~min}$ and then washed 3 times with phosphate buffer solution (PBS). Subsequently, the ER / PR primary specific monoclonal antibody produced by Novocastra was applied to the slides and after incubation, washed three times with PBS, then the secondary antibody was added. After each step, slides were washed three times with PBS. Streptavidin conjugated with HRP enzyme that can oxidize diaminobenzidine (DAB) was used to stain cells [18-20]. Finally, the slides were examined using an optical microscope equipped with an advanced camera plus 2 and images were taken. It should be noted that these slides were examined separately by two pathologists. After staining, cells with less than 5\% staining were considered negative and those with more than 5\% brown spots were considered positive [12]. Samples with at least one type of positive receptor (ER + / PR-, ER- / PR + or ER +/ PR +) are considered positive and samples with ER- / PR-phenotype were considered negative and no gene expression.

\section{Statistical analysis}

Data were analyzed using SPSS 16 software and Fisher, s exact and chi-square tests were used if necessary. Values with p-value less than or equal to 0.05 were considered as significant difference. 


\section{Results}

Relationship between pathological and histological findings with grade and stage of tumors

The minimum age in this study was 25 and up to 80 years. Of 74 malignancy samples, $61(82.4 \%)$ were invasive ductal carcinoma and $13(17.6 \%)$ were non-invasive ductal carcinoma. 15 (20.3\%) patients were carcinoma in situ, meaning that the cancer cells were confined to a single lobule and duct. They were not infected with adipocytes around the cancer cells. 10 cases $(13.5 \%)$ were in stage I of progression of the disease that the lymph nodes were not exposed to the cancer cells. 23 samples (31.1\%) were lymph nodes adjacent to the malignant cells. They were infected, meaning they were in stage II (stage II) of disease progression. 16 samples (21.6\%) were in stage 3 (Stage III) disease and 10 samples $(13.5 \%)$ were in the stage of malignancy, meaning they had metastasis to other tissues (Stage IV). There were not any significant differences between tumor stage and different ER / PR phenotypes ( $\mathrm{P}=0.36$, Table 1$)$.

14 cases $(18.9 \%)$ were in grade one and 41 cases (55.4\%) in grade two and 19 cases (25.7\%) in grade three in terms of histological and pathological progression (Table 2). There was no significant difference between Grades with the expression of different ER / PR phenotypes $(\mathrm{P}=0.38)$.

Relationship between different $E R / P R$ phenotypes with invasive and non-invasive tumor types

From 74 samples, $73(98.6 \%)$ had at least one positive ER / PR phenotype and one (1.4\%) had negative ER / PR phenotype. From 73 positive samples: $47(63.5 \%)$ were ER + / PR + phenotype, 35 of which (47.3\%) were invasive ductal tumors and $12(16.2 \%)$ were non-invasive ductal tumors. 6 (8.1\%) were ER + / PR - phenotype samples. There were $5(6.8 \%)$ in invasive ductal tumors group and $1(4.1 \%)$ was in the non-invasive ductal tumors group. $20(27 \%)$ were ER - / PR + and all of them were in invasive ductal tumors group (Table 3). One sample had ER- / PR- phenotype, which was in the invasive ductal tumors group. There was not detected ER- / PRphenotype in non-invasive tumor $(\mathrm{P}=0.88)$.

Relationship between premenopausal and menopausal age with different $E R / P R$ expression phenotypes

The mean age of patients with at least one ER / PR-positive phenotype was $54 \pm 12.39$ years, while the

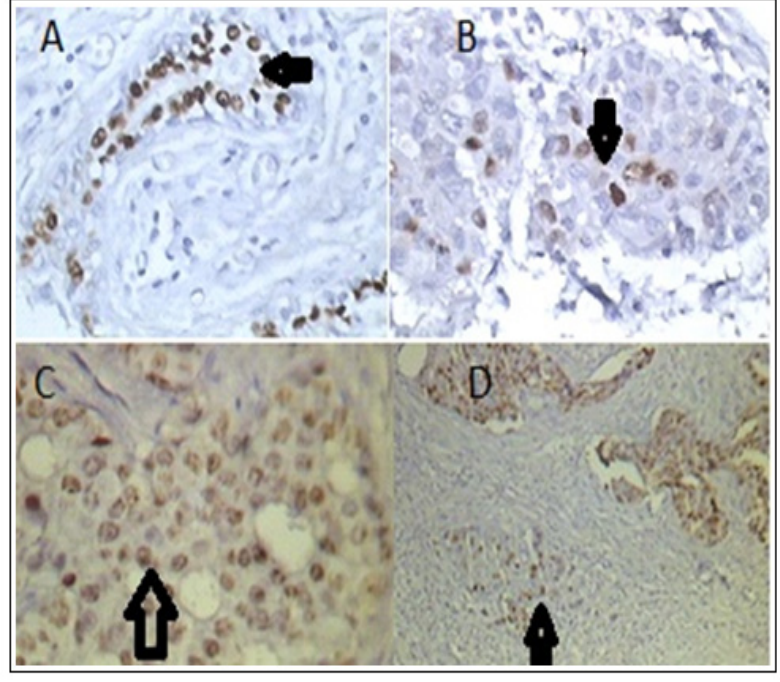

Figure 1. Cross-sectional Area of Cells Stained by Immunohistochemistry Method in 4 Micron Samples of Normal and Cancerous Breast Tissue. Figure A, Arrow shows $\mathrm{PR}+$ in normal breast tissue. Figures $\mathrm{B}$ and $\mathrm{C}$, arrows show ER + in cancerous cells of breast cancer tissue ( $\times 400$ magnification). Figure D, Arrow show $\mathrm{PR}+$ in cancerous cells of breast cancer tissue $(\times 100$ magnification).

age of ER-/PR- phenotype was 53 years. There was no statistically significant difference between the age of premenopausal and menopause with ER / PR phenotype $(\mathrm{P}=0.61)$.Control (healthy) samples were positive for ER / PR phenotype in terms of expression of hormone receptors (Figure 1).

There was not detected expression of ER- / PRphenotype in non-invasive ductal tumors. There was not a significant difference between invasive and non-invasive ductal tumors with expression of different phenotype of $\mathrm{ER} / \mathrm{ER}$ hormone receptors in breast cancer $(\mathrm{P}=0.088)$.

\section{Discussion}

This descriptive-analytical study was performed to investigate the relationship between different ER / PR phenotypes with tumor type and histopathologic markers in breast cancer malignancy. From the 74 malignant samples, 61 were invasive ductal carcinoma and 13 were non-invasive ductal carcinoma. There was an increase in phenotype of positive ER / ER hormone receptor in non-invasive ductal tumors compared to other tumor

Table 1. Relationship between Pathological and Histological Findings with Stage of Tumors

\begin{tabular}{lccccc}
\hline Phenotype & ER+/PR+ & ER+/PR- & ER-/PR+ & ER-/PR- & Total \\
\hline Stages & Frequency & Frequency & Frequency & Frequency & Frequency \\
Stage zero & $10(66.7 \%)$ & $2(13.3 \%)$ & $3(20.0 \%)$ & $0(0.0 \%)$ & $15(100.0 \%)$ \\
Stage 1 & $7(70.0 \%)$ & $0(0.0 \%)$ & $2(20.0 \%)$ & $1(10.0 \%)$ & $10(100.0 \%)$ \\
Stage 2 & $13(56.5 \%)$ & $1(4.3 \%)$ & $9(39.1 \%)$ & $0(0.0 \%)$ & $23(100.0 \%)$ \\
Stage 3 & $12(75.0 \%)$ & $2(12.5 \%)$ & $2(12.5 \%)$ & $00(0.0 \%)$ & $16(100.0 \%)$ \\
Stage 4 & $5(50.0 \%)$ & $1(10.0 \%)$ & $4(40.0 \%)$ & $00(0.0 \%)$ & $10(100.0 \%)$ \\
Total & $47(63.5 \%)$ & $6(8.1 \%)$ & $20(27.0 \%)$ & $1(1.4 \%)$ & $74(100.0 \%)$ \\
\hline
\end{tabular}


Table 2. Relationship between Pathological and Histological Findings with Tumors Grade

\begin{tabular}{lccccc}
\hline Phenoty pe Grade & ER+/PR+ & ER+/PR- & ER-/PR+ & ER-/PR- & Total \\
\hline Grade 1 & $7(50.0 \%)$ & $1(7.1 \%)$ & $6(42.9 \%)$ & $0(0.0 \%)$ & $14(100.0 \%)$ \\
Grade 2 & $30(73.2 \%)$ & $2(4.9 \%)$ & $8(19.5 \%)$ & $1(2.4 \%)$ & $41(100.0 \%)$ \\
Grade 3 & $10(52.6 \%)$ & $3(15.8 \%)$ & $6(12.5 \%)$ & $00(0.0 \%)$ & $19(100.0 \%)$ \\
Total & $47(63.5 \%)$ & $6(8.1 \%)$ & $20(27.0 \%)$ & $1(1.4 \%)$ & $74(100.0 \%)$ \\
\hline
\end{tabular}

Table 3. Relationship between Different ER / PR Phenotypes with Invasive and Non-invasive Tumor Types

\begin{tabular}{lccccc}
\hline Phenotype & ER+/PR+ & ER+/PR- & ER-/PR+ & ER-/PR- & Total \\
\hline Tumor Types & & & & & \\
Invasive Carcinoma & $35(47.3 \%)$ & $5(6.8 \%)$ & $20(27.0 \%)$ & $1(1.4 \%)$ & $61(82.4 \%)$ \\
Non Invasive Carcinoma & $12(16.2 \%)$ & $1(1.4 \%)$ & $0(0.0 \%)$ & $0(0.0 \%)$ & $13(17.6 \%)$ \\
Total & $47(63.5 \%)$ & $6(8.1 \%)$ & $20(27.0 \%)$ & $1(1.4 \%)$ & $74(100.0 \%)$ \\
\hline
\end{tabular}

type. Also, it was not detected expression of ER- / PRphenotype in non-invasive ductal tumors.

In a study conducted by Stolnicu $\mathrm{S}$ et al on 112 breast cancer patients, $75 \%$ of the samples in that study were positive for ER $+/ \mathrm{PR}+$ receptors [21).In the present study, $47(63.5 \%)$ of non-invasive and invasive ductal carcinoma specimens had positive ER + / PR + phenotype. The present study is largely consistent with the above study regarding the expression of these positive hormone receptor phenotypes in breast cancer. In a study conducted by Zhang L et al, there was a direct link between expression of ER $+/ \mathrm{PR}+$ receptors and the response to chemotherapy in breast cancer patients, so careful evaluation of these markers is essential in the therapeutic process [22]. Rauscher GH et al reported that there was no significant relationship between ER $+/ \mathrm{PR}+$ and tumor stage, mortality, and survival [16], Our findings are consistent with that and there is not significant difference between the tumor stages with each of the different ER / PR phenotypes. On the other hand, the study of Gahlaut $R$ et al shows that there is a statistically significant relationship between grade and expression of hormonal receptors [15], while in present study there was no statistically significant association between grade and different ER / PR phenotypes in breast cancer. This difference reflects the fact that expression of different ER / PR phenotypes of hormone receptors with grade characteristic tumors in all societies do not naturally follow a completely identical pattern, most likely to have other factors other than grade and stage. It can vary from one geographical location to another.

In the present study, the negative phenotype of hormone receptors, ER- / PR-, was not detected in non-invasive ductal tumors, whereas this negative phenotype was only seen in the invasive ductal tumors. This isthe highlight of this study. Why the ER- / PRnegative phenotype was observed only in the invasive ductal tumor? A precise answer to this question requires further studies in other geographical areas. However, it is possible that the tumor type may also be involved in the expression of this negative hormone phenotype ER-/ PR-.

Since breast cancer is the second fatal cancer in women in the world, it seems it is necessary to process, diagnose, follow-up therapy, and prognosis not only positive hormonal phenotypes such as $\mathrm{ER}+/ \mathrm{PR}+$, we must also consider the type of tumor, i.e., invasive or non-invasive ductal tumorsas an effective factor.

On the other hand, it is also possible to think that in ER- / PR-negative phenotype, non-expression of other genes, such as $\mathrm{p} 53$, which downregulate the promoter or expression of other genes may directly or indirectly contribute to this phenomena [7]. Therefore, it should not be missed or neglected that the initial diagnostic value of the typing of ductal tumors (invasive or non-invasive tumors) by skill and experience of the oncologists in visual testing and with the help of markers. In another study, Dibaba DT et al showed that there are other factors, such as metabolic syndrome, immobility risk, and obesity that do not reduce the risk of mortality even in patients with $\mathrm{ER}+/ \mathrm{PR}+$ hormone receptor phenotype[23]. However, risk factors, such as immobility and other unknown factors, or high age of postmenopausal, increase the risk of mortality. Also, Exercise and mobility exercises have been reported to partially relieve fatigue in patients with breast cancer [24]. There was not statistically significant relationship between different ER / PR phenotypes and age at menopause and premenopause.

In conclusion, ER- / PR-phenotype only observed in invasive ductal tumor. It is thought that the tumor type may affect the expression of different types of ER / PR hormone receptor phenotypes in breast cancer patients.

\section{Acknowledgments}

We would like to thank Mrs. Mahmoudi, Expert in Pathology Laboratory and Dr. Rakhshani and Dr. Jalilian who were part of this project.

\section{References}

1. Lam WWT, Fielding R, Ho EYY. Predicting psychological morbidity in Chinese women after surgery for breast carcinoma. Cancer. 2005;103(3):637-646. https://doi. org/10.1002/cncr.20810

2. Naghavi M, Abajobir AA, Abbafati C, Abbas KM, AbdAllah F, Abera SF, Aboyans V, et al. Global, regional, and 
national age-sex specific mortality for 264 causes of death, 1980-2016: a systematic analysis for the Global Burden of Disease Study 2016. The Lancet. 2017 09;390(10100):11511210. https://doi.org/10.1016/s0140-6736(17)32152-9

3. Sharma R. Breast cancer incidence, mortality and mortalityto-incidence ratio (MIR) are associated with human development, 1990-2016: evidence from Global Burden of Disease Study 2016. Breast Cancer. 201901 02;26(4):428445. https://doi.org/10.1007/s12282-018-00941-4

4. Jazayeri SB, Saadat S, Ramezani R, Kaviani A. Incidence of primary breast cancer in Iran: Ten-year national cancer registry data report. Cancer Epidemiology. 2015 08;39(4):519-527. https://doi.org/10.1016/j.canep.2015.04.016

5. Akbarzadeh R, Fatemeh Ghardashi, Rasool Tabari, Akram Koshki, Masoumeh Hashemiyan, Tayyebeh Novrozinejad, Hamid Alami. Individual characteristics, family history and blood group in with breast cancer in Sabzevar. Journal of Sabzevar University of Medical Sciences. 2014;20(5):582-589. https://www.sid.ir/en/Journal/ ViewPaper.aspx?ID=370217.

6. Kumar V, Robbins S. Chapter. Robbins Basic Pathology., Robbins Basic Pathology, 8 th Edition, Philadelphia, PA. Saunders/Elsevier: 2007.

7. Syarif H, Waluyo A, Afiyanti Y, Mansyur M. Executive function in breast cancer survivors and the influencing factors. Enfermería Clínica. 2019 09;29:280-285. https:// doi.org/10.1016/j.enfcli.2019.04.034

8. Golmohammadi R, Pejhan A. The prognostic value of the P53 protein and the Ki67 marker in breast cancer patients. J Pak Med Assoc. 2012;62(9):871-875. PMID: 23139965.

9. Cui C, Li S, Wu D. Znhit1 inhibits breast cancer by upregulating PTEN to deactivate the $\mathrm{PI} 3 \mathrm{~K} / \mathrm{Akt} / \mathrm{mTOR}$ pathway. Life Sciences. 2019 05;224:204-211. https://doi. org/10.1016/j.1fs.2019.03.067

10. Golmohammadi R, Rakhshani MH, Moslem AR, Pejhan A. Prognostic Role of PTEN Gene Expression and Length of Survival of Breast Cancer Patients in the North East of Iran. Asian Pacific Journal of Cancer Prevention. 201606 01;17(sup3):305-309. https://doi.org/10.7314/ apjcp.2016.17.s3.305

11. Abdeen SK, Aqeilan RI. Decoding the link between WWOX and p53 in aggressive breast cancer. Cell Cycle. 201905 16;18(11):1177-1186. https://doi.org/10.1080/15384101.2 019.1616998

12. Miles J, White Y. Neratinib for the Treatment of Early-Stage HER2-Positive Breast Cancer. Journal of the advanced practitioner in oncology. 2018;9(7):750. PMID: 31249722 PMCID: PMC6570523.

13. Korobeynikov V, Borakove M, Feng Y, Wuest WM, Koval AB, Nikonova AS, Serebriiskii I, Chernoff J, Borges VF, Golemis EA, Shagisultanova E. Combined inhibition of Aurora A and p21-activated kinase 1 as a new treatment strategy in breast cancer. Breast Cancer Research and Treatment. 201906 28;177(2):369-382. https://doi. org/10.1007/s10549-019-05329-2

14. Lim E, Tarulli G, Portman N, Hickey TE, Tilley WD, Palmieri C. Pushing estrogen receptor around in breast cancer. Endocrine-Related Cancer. 2016 Dec;23(12):T227-T241. https://doi.org/10.1530/erc-16-0427

15. Gahlaut R, Bennett A, Fatayer H, Dall BJ, Sharma N, Velikova G, Perren T, Dodwell D, Lansdown M, Shaaban AM. Effect of neoadjuvant chemotherapy on breast cancer phenotype, ER/PR and HER2 expression - Implications for the practising oncologist. European Journal of Cancer. 2016 06;60:40-48. https://doi.org/10.1016/j.ejca.2016.03.006

16. Rauscher GH, Silva A, Pauls H, Frasor J, Bonini MG,
Hoskins K. Racial disparity in survival from estrogen and progesterone receptor-positive breast cancer: implications for reducing breast cancer mortality disparities. Breast Cancer Research and Treatment. 201703 01;163(2):321-330. https://doi.org/10.1007/s10549-017-4166-z

17. Shet $T \mathrm{~T}$. Improving accuracy of breast cancer biomarker testing in India. The Indian journal of medical research. 2017;146(4):449. https://doi.org/https://doi.org/10.4103/ ijmr.IJMR $896 \quad 16$

18. Schrijver WAME, van der Groep P, Hoefnagel LD, ter Hoeve ND, Peeters T, Moelans CB, van Diest PJ. Influence of decalcification procedures on immunohistochemistry and molecular pathology in breast cancer. Modern Pathology. 201608 26;29(12):1460-1470. https://doi.org/10.1038/ modpathol.2016.116

19. Zaha DC. Significance of immunohistochemistry in breast cancer. World Journal of Clinical Oncology. 2014;5(3):382. https://doi.org/10.5306/wjco.v5.i3.382

20. Plichta JK, Ren Y, Thomas SM, Greenup RA, Fayanju OM, Rosenberger LH, Hyslop T, Hwang ES. Implications for Breast Cancer Restaging Based on the 8th Edition AJCC Staging Manual. Annals of Surgery. 2020 01;271(1):169176. https://doi.org/10.1097/sla.0000000000003071

21. Stolnicu S, Bauer O, Naznean A, Podoleanu C, Georgescu R. ER-/PR+ subset of invasive breast carcinoma (IBC): a distinct phenotype with good prognosis. Polish Journal of Pathology. 2018;69(3):311-313. https://doi.org/10.5114/ pjp.2018.79551

22. Zhang L, Yu Q, Wu X, Hsieh M, Loch M, Chen VW, Fontham E, Ferguson T. Impact of chemotherapy relative dose intensity on cause-specific and overall survival for stage I-III breast cancer: ER+/PR+, HER2- vs. triple-negative. Breast Cancer Research and Treatment. 201801 24;169(1):175-187. https://doi.org/10.1007/s10549-017-4646-1

23. Dibaba DT, Ogunsina K, Braithwaite D, Akinyemiju T. Metabolic syndrome and risk of breast cancer mortality by menopause, obesity, and subtype. Breast Cancer Research and Treatment. 2018 Nov 21;174(1):209-218. https://doi. org/10.1007/s10549-018-5056-8

24. Juvet L, Thune I, Elvsaas I, Fors E, Lundgren S, Bertheussen $\mathrm{G}$, Leivseth G, Oldervoll L. The effect of exercise on fatigue and physical functioning in breast cancer patients during and after treatment and at 6 months follow-up: A meta-analysis. The Breast. 2017 06;33:166-177. https://doi.org/10.1016/j. breast.2017.04.003

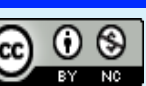

This work is licensed under a Creative Commons AttributionNon Commercial 4.0 International License. 